\title{
Position and strength optimization for secondary source of transformer active noise control system
}

\author{
Wang Guodong ${ }^{1, a}$, Ying Liming ${ }^{1, b}$, Liu Yang ${ }^{2}$, Chang Yong ${ }^{1}$, Wang Jinwei ${ }^{1}$, \\ Yang Peng ${ }^{1}$
}

${ }^{1}$ School of Electrical Engineering, Wuhan University, Wuhan 430072, Hubei Province, China

${ }^{2}$ Maintenance Company, State Grid Hubei Electric Power Company, Wuhan 430050, China

aiguodong2009@163.com, 'Imying@whu.edu.cn

Keywords: active noise control; active quiet zone in local space; secondary sound source; position and strength

\begin{abstract}
The choice of the parameters of the secondary sound source which contain the number、 position and strength is a key part in the design of active noise control system of power transformer. Countering the practical applications environment of noise control of transformer, acoustic radiation model in half-free space is established. After selecting the sum of the squared sound pressures of the observation points as the objective function, the genetic algorithm is used to optimize the position of secondary sound source and the optimal source intensity can be resolved by using the analytical method. By single frequency experiment, the availability of applying optimization scheme of the secondary sound sources to practical transformer noise control is verified.
\end{abstract}

\section{Introduction}

With the rapid development of the national economy and the city extensions, the high voltage transmission line is introduced into the urban area, and many large capacity and high voltage power transformers are also entered into the urban area. The noise generated by the normal operation of power transformer will have a serious impact on the daily life and rest of the nearby residents, so people pay more and more attention to the noise pollution caused by this problem[1]. The problem of noise control is becoming more and more important.

In principle, active noise control (ANC) can be viewed as the superposition of a secondary sound field, generated by the control system, on a primary, disturbing sound field such that the residual sound field is as small as possible. So, basically, the objective of the active control system is to generate a secondary sound field that matches the primary sound field as closely as possible, both in time and in space ${ }^{[2]}$. Compared with the traditional passive technology, active noise control technique is especially suitable for low frequency noise ${ }^{[3]}$. An active noise control system is mainly divided into physical and electronic part. The purpose of the physical design is to determine the number of secondary sound sources and its locations and strength in the acoustic field. The choice of the parameters of the secondary sound source not only affects the stability of the whole control system, but also affects the control effect of the noise ${ }^{[4]}$.A great amount of literature has focused on the control strategies or algorithms, but little research has been conducted in addressing the optimized design of the ANC physical system.

In practical engineering applications, there are only some areas have the requirement of noise control such as residential building near the substation. In order to achieve the purpose of local spatial noise cancellation, Mazzanti ${ }^{[5]}$ proposed to use the array type secondary source to achieve the purpose of local spatial noise reduction.

In paper[6], the influence of the relative parameters of the secondary sound source array, such as the number of the secondary sound source, the distance between the secondary sound source, and the distance between the secondary sound source and the noise source, is analyzed in theory, and the numerical simulation is carried out. The simulation studies the influence of the relative parameters by a single variable method. A multi-level sub array active control theory is proposed and applied to the local spatial control of the low frequency noise in[7]. In this paper, the influence of the number 
of secondary sound sources and the spatial position of the secondary sound source is obtained by calculation and simulation based on the noise data of the transformer. In paper [8-9], a typical control arrangement with multiple secondary sources and multiple error sensors equally spaced in two parallel lines is proposed and results from computer simulation and experiments in an anechoic chamber show that there exists a range of optimal spacing for the secondary sources and error sensors. But the primary sound source can't simply be considered as a point sound source, so it is difficult to achieve the design goal in the practical engineering applications.

Through referring existed literature, being aimed to the application environment of transformer noise cancellation, the optimization parameter of different number secondary source is obtained by the combination method of analytical method and Genetic algorithm. Experimental results verify the effectiveness of the optimization scheme.

\section{Acoustic radiation model of transformer noise field}

\section{Model of primary and secondary sound sources}

Given that the noise of power transformer radiated mainly by surface outward, and the size of power transformer is close to the half wavelength of acoustic wave, the radiation model of power transformer can be simplified to the model of the pulse spherical sound source neglecting the harmonic time factor $e^{j \omega t}$.And the radiation pressure can be expressed as Eq. 1:

$$
\left\{\begin{array}{l}
p_{p}=\frac{|\boldsymbol{A}|}{r-r_{0}} e^{j\left[-k\left(r-r_{0}\right)+\theta\right]} \\
|\boldsymbol{A}|=\frac{\rho_{0} c_{0} k r_{0}^{2} \mu_{a}}{\sqrt{1+\left(k r_{0}\right)^{2}}} \\
\theta=\arctan \frac{1}{k r_{0}}
\end{array}\right.
$$

Where $p_{p}$ is sound pressure of primary source at observation point, $r$ is the distance between the observation points and spherical center, $r_{0}$ is radius of the sphere. $A$ is complex numbers, $|A|$ is its amplitude, $q$ is its phase angle. $r_{0}$ is air density, $c_{0}$ is the propagation velocity of sound waves in the air, $\mu_{a}$ is the amplitude of the vibration velocity of the spherical source, $k$ is wave number and $k=\omega / c_{0}$.

In addition, because speaker is usually chosen as the secondary sound source in the active noise control system, its characteristic scale is negligible compared to the half wavelength of the sound wave, so the secondary sound source is approximately equivalent to the point sound source model, and the radiation pressure is given by Eq. 2:

$$
p_{s}=\frac{j k \rho_{0} c_{0} q_{s}}{4 \pi r} e^{-j k r}
$$

Where $p_{s}$ is sound pressure of secondary sound source at observation point, $r$ is the distance between the observation points and point sound source, $q_{s}$ is intensity of secondary sound source.

\section{Acoustic radiation model transformer noise in the half free space}

If the ground surface is a reflection surface of space field, and the other directions are in accord with the condition of free space field, so this space field is often referred to half free space. Half free space acoustic field is a kind of sound field environment which is more suitable for the transformer. Radiated acoustic field of point sound source in the half free space can, by image theory, be equivalent to superimposed sound field of itself and its mirror image(also referred as virtual source), it can be shown in Fig. 1. 


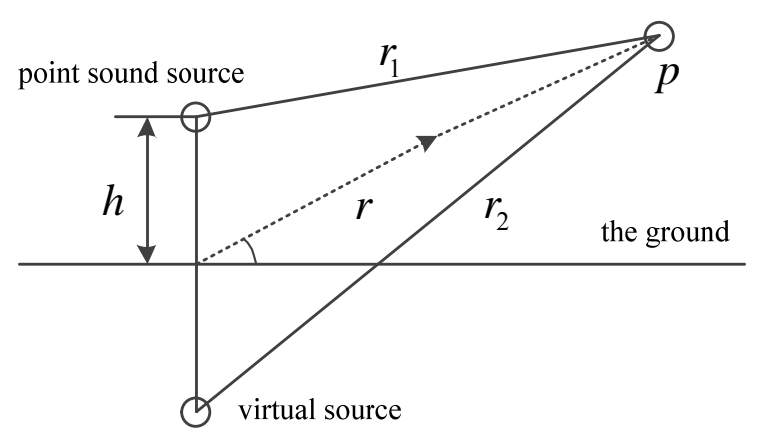

Fig. 1 Acoustic radiation model of point sound source in half free space

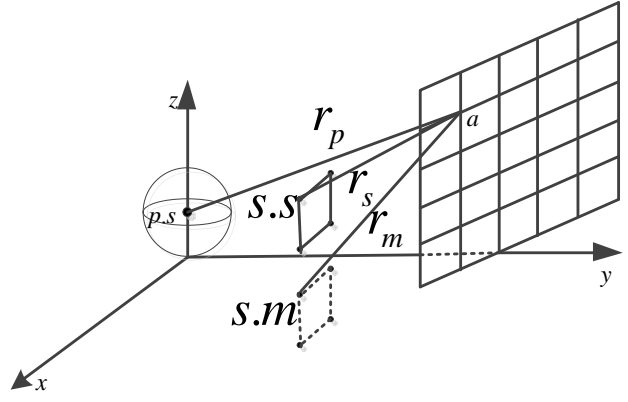

Fig. 2 Active noise control model in half free space

The sound pressure of point sound source in half free space at the observation point $\mathrm{P}$ can be given by:

$$
p=\frac{A}{r_{1}} \mathrm{e}^{j\left(\omega t-k r_{1}\right)}+\eta \frac{A}{r_{2}} \mathrm{e}^{j\left(\omega t-k r_{2}\right)}
$$

Where $r_{1}$ and $r_{2}$ are the sound source, the virtual source to the observation point of the distance, respectively, $\eta$ is the sound reflection coefficient of rigid wall.

Suppose a power transformer is located in the half free space field in which secondary source array comprised of $\mathrm{N}$ speaker is used to cancel the noise produced by the transformer and there is a noise plane to be cancelled in the far field. According to the superposition principle of sound waves, the acoustic pressure $p_{a}$ at observation point $a$ in local area is:

$$
p_{a}=p_{p}+Z_{r} q_{s}
$$

Where $p_{p}$ is acoustic pressure of primary sound source at point $a, q_{s}=\left(q_{s 1}, q_{s 2}, \cdots, q_{s N}\right)^{T}$ is a $N \times 1$ vector of intensity of $\mathrm{N}$ speakers is a $N \times 1$ column vector, $Z_{r}$ is row vector of the sum of acoustic transfer impedance from secondary sources and virtual source to the observation position $a$, $Z_{r}$ can be expressed as

$$
Z_{r i}=\frac{j k \rho_{0} c_{0}}{4 \pi r_{s i}} e^{-j k r_{s i}}+\eta \cdot \frac{j k \rho_{0} c_{0}}{4 \pi r_{\text {smi }}} e^{-j k r_{s m i}}
$$

Where $r_{s i}$ and $r_{s m i}$ are the distance from secondary source and its virtual source to the observation point $a$, and $\eta$ is the acoustic reflection coefficient of the ground.

Similarly, the acoustic pressure of the $\mathrm{M}$ observation points, which is chosen from the noise plane to be cancelled, can be expressed as:

$$
P_{M}=P_{p}+Z_{M r} q_{s}
$$

Where $p_{M}$ is the column vector of the acoustic pressure of $\mathrm{M}$ observation points, $P_{p}=\left(P_{p 1}, P_{p 2}, \cdots, P_{p M}\right)^{T}$ is the $M \times 1$ column vector of sound pressures at the $\mathrm{M}$ observation points due to primary source. $Z_{M r}$ is the $M \times N$ matrix of acoustic transfer impedance from secondary sources to the $\mathrm{M}$ observation points and can be given by:

$$
Z_{r i}=\frac{j k \rho_{0} c_{0}}{4 \pi r_{s i j}} e^{-j k r_{s i j}}+\eta \cdot \frac{j k \rho_{0} c_{0}}{4 \pi r_{s m i j}} e^{-j k_{s m i j}}
$$

Where $r_{s i j}$ and $r_{\text {smij }}$ are the distance of any secondary source $i$ and its virtual source to the number $\mathrm{j}$ of the observation point, respectively.

The sum of the squared sound pressures of the M points is chosen as the objective function of the control system

$$
\begin{aligned}
J\left(q_{s}\right) & =p_{M}^{H} p_{M} \\
& =q_{s}^{H} Z_{r}^{H} Z_{r} q_{s}+p_{p}^{H} Z_{r} q_{s}+q_{s}^{H} Z_{r}^{H} p_{p}+p_{p}^{H} p_{p}
\end{aligned}
$$


Where $J\left(q_{s}\right)$ is quadratic function of the intensity of secondary sound source. By minimization the objective function, the optimal source intensity can be resolved:

$$
q_{s 0}=-\left(Z_{r}^{H} Z_{r}\right)^{-1} Z_{r}^{H} P_{p}
$$

So with $\boldsymbol{q}_{s o}$, the acoustic pressure of the M points can be expressed as:

$$
P_{M \min }=P_{p}+Z_{r} q_{s o}
$$

In order to assess achievable attenuation with this distribution of secondary sources in the plane, another $N$ monitoring points are chosen to calculate the attenuation level(AL), and the AL can be expressed as:

$$
A L=10 \log _{10} \frac{\left|p_{p i}\right|}{\left|p_{M i}\right|},(i=1,2, \ldots, M)
$$

Where $p_{p i}$ and $p_{M i}$ are the acoustic pressure pre-and post-adding secondary source respectively. The optimized scheme can be evaluated by the rough approximation of distribution of noise reduction which can be described according to attenuation level of the $K$ monitoring points.

By analysis of the radiation model of sound field, Eq. 4 and 6 are directly related to the distance which is closely linked with the locations of the secondary sound sources. So, in this paper, genetic algorithm is used to optimize the Position of secondary sources with different number, and then the optimal source intensity can be calculated by Eq. 9. Through the optimization of the secondary source parameter, active noise reduction system could minimize the noise level in the local plane.

\section{Optimization of the secondary source parameter}

The sketch of active noise control model of transformer is shown in Fig.3. Reflective surface of ground is chosen as the plane $x o y$ of the 3D Cartesian coordinate system. For practical purposes that primary sound source and secondary sound source array are all on the ground; This paper simplify the power transformer to the model of the pulse spherical sound source, and we set its center at $(0,0,2)$ as the radius of the primary source $r_{0}=2 \mathrm{~m}$, the amplitude of vibration velocity of the transformer surface approximately $u_{a}=6.3 \times 10^{-4}$; The secondary source array is parallel to the plane $X O Z$, and the geometric center of the secondary source array and the center of the sphere model are on the same line; The secondary source array and its image are symmetric about the plane $x o y$; a $10^{\prime} 10 \mathrm{~m}$ plane that is parallel to the plane of secondary source array is chosen as the noise reduction plane which is $10 \mathrm{~m}$ away from the center of the primary source, the plane is divided into a $11 \times 11$ grid and 121 observation points is obtained; Air density and sound velocity are $\rho_{0}=1.29$ $\mathrm{kg} / \mathrm{m}^{3}, c_{0}=344 \mathrm{~m} / \mathrm{s}$ respectively.

The sum of the squared sound pressures of the M points is chosen as the objective function of the control system which can be optimized by the genetic algorithm. The parameter of genetic algorithm can be set as follows: the population size is 100 and crossover probability is $P_{c}=1.0$; Uniform selection function is chosen as the variogram function and its variation probability is $P_{m}=0.01$; The maximum number of iterations is 100 times. Each combination of secondary source would be optimized 10 times in relation to different number of speaker and then take an average as the final optimization results. And three parameters are chosen to be optimized: (1)The distance $r_{p s}$, between secondary source array and the center of the primary source which is greater than the radius of the pulse spherical sound source, that is $r_{p s}>2 ;(2)$ horizontal space between two adjacent secondary source, $r_{\text {ss } 1}(3)$ longitudinal space between two adjacent secondary source, $r_{s s 2}$. Finally, the optimization results is shown as Fig. 3: 


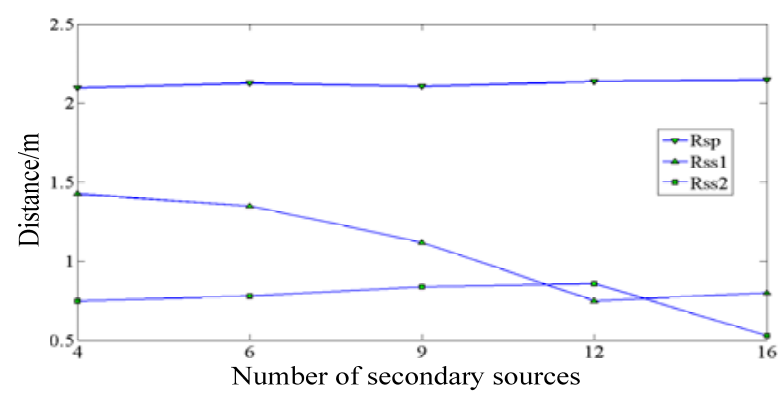

Fig.3 The optimization results

Some conclusions have been drew from Fig. 3: (1) whatever the number of secondary source is, the secondary sources array should try to be close to primary sources. (2)The distances of the horizontal and longitudinal space of two adjacent secondary sources are gradually decreasing as the increasing number of secondary source.

\section{Experiment on optimizing the parameter of the secondary source}

\section{Experimental conditions}

To verify the validity and utility of the optimization scheme obtained from above section, our team performed an experiment. Experimental block diagram is shown in Fig. 4. Signal generator send out the single-frequency signal to drive the loudspeakers, combined with the method of low-frequency noise simulation and equivalence of power transformer proposed by Zhang[10], we could simulate actual noise level of substation by adjusting phase and amplitude of the signal which also input to industrial computer; The industrial computer calculates the secondary signal that is sent to drive the secondary sound source array; The noise level of the experimental site is measured by the noise meter.

Our experiment performed in an open spaces which almost matched with the practical applications environment of noise control of transformer; in this paper, we choose two speaker to simulate the primary source; the secondary source array is parallel to the primary source; the noise plane to be reduced is $10 \mathrm{~m}$ away from the primary source; To evaluate the performance of the optimization scheme, we choose A,B and $\mathrm{C}$ group of 15 points in the upper, middle and lower of the noise plane respectively to measure the noise reduction level, and both horizontal space and longitudinal space between two adjacent measuring points are $1 \mathrm{~m}$. The sketch of experimental site is shown in Fig.5.

The experiment only certify the validity of optimization scheme, both position and strength which can be resolved by Eq. 9, with 4, 6 and 9 secondary source. In order to evaluate the effectiveness of the optimization scheme directly, the corresponding contrasted experiments are also performed. And the concrete setting is: the distance between primary source and the secondary source array is $1.7 \mathrm{~m}$; When the number of the secondary source is 4, horizontal space and longitudinal space between two adjacent are $0.7 \mathrm{~m}$ and $1.2 \mathrm{~m}$ respectively; When the number of the secondary source is 6 , horizontal space and longitudinal space are $0.6 \mathrm{~m}$ and $0.4 \mathrm{~m}$ respectively; and when the number is 9 , horizontal space and longitudinal space are 0.6 and $0.4 \mathrm{~m}$ respectively.

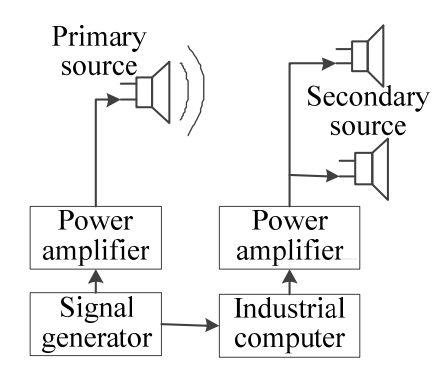

Fig. 4 Experimental block diagram

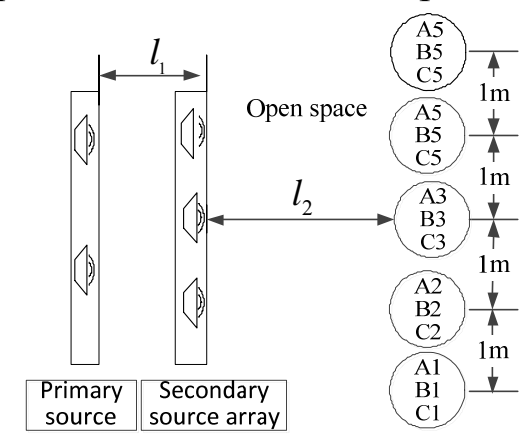

Fig. 5 Sketch of experimental site 


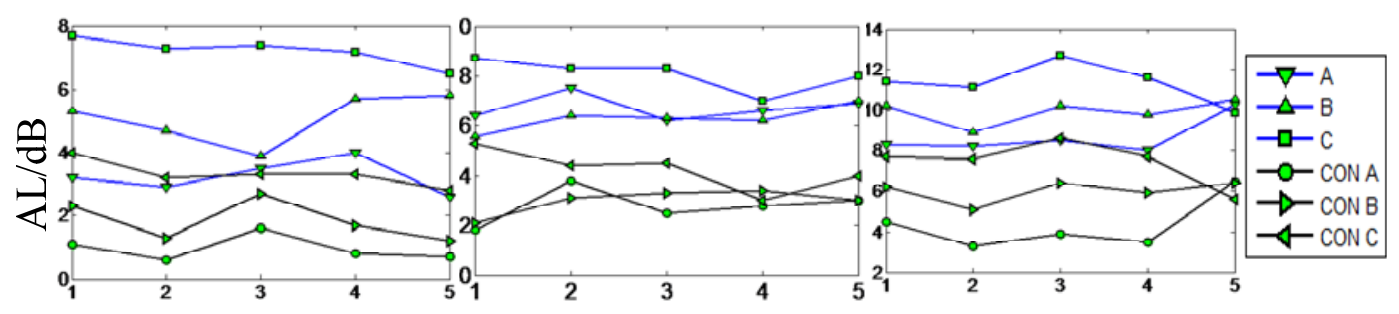

4 secondary source 6 secondary source 9 secondary source

Fig. 6 Noise reduction of the monitoring points

\section{Experimental results and analysis}

First, open signal generator and measure the A-weighted sound pressure level of $100 \mathrm{~Hz}$ single-frequency at monitoring points; Then, adjust the location and strength of the secondary source according to the optimization scheme obtained in last section, and open it; Finally, measure the A-weighted sound pressure level of $100 \mathrm{~Hz}$ single-frequency at monitoring points once again. Experimental results are shown in Fig.6. Where CON is represent the contrasted group.

Experimental results confirm that the more close to the geometric center of the noise, the more close to the ground, the larger the amount of noise reduction is obtained. And the noise reduction of the monitoring point show that compared with the empirical scheme, the optimization scheme has better noise control effect.

\section{Summary and conclusions}

Position and strength of the secondary source not only affect the stability of the whole control system, but also affect the control effect of the noise. Being aimed to the need of transformer noise control, the optimization schemes with different number of secondary source are obtained using the method proposed in this paper that combined the genetic algorithm with analytical method. And some laws of position selection are summarized in this paper. In order to verify the validity of the optimization scheme, our team performed some single-frequency experiments in which simulated the actual noise level of transformer substation, and a plane which is $10 \mathrm{~m}$ away from the primary source is chosen as the noise plane to be cancelled. And our team also set up a contrast experiment with empirical scheme in which location and the corresponding strength of secondary source are different from the optimization scheme. Through measure the noise level of monitoring points, the effectiveness of the optimization scheme is verified by the considerable amount of noise reduction.

\section{Acknowledgments}

Project supported by the National Natural Science Foundation of China (No. 51377123).

\section{References}

[1] Jiangtao Liu, Liming Ying. Research on active noise control in power transformer[J]. Advanced Materials Research, 2011, 347-353:2347-2350.

[2] Xiaojun Qiu, Xun Li, Yanting Ai, et al. A waveform synthesis algorithm for active control of transformer noise: implementation[J]. Applied Acoustics, 2002, (63):467-79.

[3] Bing Li, Guoqing Hu. Discussion on measure of reduction of transformer noise[J]].Transformer, 2004, 41(8):40-42.

[4] Xun Li. Physical system design for the active control of electrical transformer noise[D]. Ph. D degree thesis, The University of Adelaide, 2000.

[5] Mazzanti S, Piraux J. An experiment of Active Noise Attenuation in Three-dimensional Space. Inter Noise, 1983:437 430

[6] Fei Han, Jiazheng Sha. Research on secondary source array in active noise attenuation in 
space[J]. Journal of Nanjing university: natural section, 1996, 32(3): 433-439.

[7] Xiyue Ma, Kean Chen, Han Hu. Layout optimization of electro-acoustic devices of active noise control system for $110 \mathrm{kV}$ transformer [J]. Noise and vibration control, 2011,06:109-115.

[8] Guo J, Pan J, Bao C. Actively created quiet zones by multiple control sources in free space[J]. The Journal of the Acoustical Society of America, 1997, 101(3): 1492-1501.

[9] Guo J, Pan J. Further investigation on actively created quiet zones by multiple control sources in free space[J]. The Journal of the Acoustical Society of America, 1997, 102(5): 3050-3053.

[10] Bin Zhang, Yaqi Zhou, Liming Ying, et al. Low-frequency noise simulation and equivalence of power transformers[J]. Engineering Journal of Wuhan University, 2014,05:642-647. 